Article

\title{
Non-Cash Risk Measure on Nonconvex Sets
}

\author{
Chang Cong ${ }^{+}(\mathbb{D})$ and Peibiao Zhao $*,+(\mathbb{D}$ \\ School of Science, Nanjing University of Science and Technology, Nanjing 210094, China; shylotte@163.com \\ * Correspondence: pbzhao@njust.edu.cn \\ + These authors contributed equally to this work.
}

Received: 6 September 2018; Accepted: 1 October 2018; Published: 1 October 2018

check for updates

\begin{abstract}
Monetary risk measures defined on a convex set are interpreted as the smallest amount of external cash that must be added to a portfolio to make the portfolio being acceptable. In the present paper, the authors introduce a new concept: non-cash risk measure, which does as a nonconvex risk measure work in a nonconvex set. In addition, the authors arrive at a convex extension of the non-cash risk measure, and offer the relationship between the non-cash risk measure and its extension.
\end{abstract}

Keywords: risk measures; non-cash measure; nonconvex sets; nonconvex measures

MSC: $91 B 30$

\section{Introduction}

Since Markowitz(1952) [1] put forward the theory of optimal portfolio selection in 1952, variance had become an influential classical financial risk measurement method. However, the mean-variance model takes the deviation of returns above the mean into account, which is obviously inconsistent with the facts. Based on the shortcomings of variance model, in 1996, Morgan J.P. [2] proposed a new risk measurement method, the VaR method, to meet the needs of its banking business and it was quickly popularized as an industry standard. But Acerbi et al. (2002) [3] and Frey et al. (2002) [4] gave the examples that VaR violates sub-additivity. This is not consistent with the basic principle that portfolio investment reduces risk.

Based on the reasons above, Artzner et al. (1999) [5] gave the definition of coherent risk measures by four axioms, and gave the representation theorem of coherent risk measures. Because of the rationality of these four axioms, the coherent risk measures were widely accepted by the risk measurement theory. Although coherent risk measures have some practical significance, in many cases, the risk of a position will increase in a non-linear manner. For example, if a position is multiplied by a large factor, an additional liquidity risk may occur. It is found that the axioms of positive homogeneity and sub-additivity in coherent risk measure are too strict, and the restrictions of positive homogeneity and sub-additivity should be relaxed. Follmer H. and Schied A. (2002) [6] extended the results of Artzner's coherent risk measures and proposed a weaker convexity axiom to replace the positive homogeneity and subadditivity. The risk measures satisfying the monotonicity, translation invariance and convexity axioms are called convex risk measures.

The risk measures above are based on complete markets, which requires no frictions in the market. However, in real life, assets transactions require payment costs. Lepinette et al. (2017) [7] studies the pricing functions when transaction costs exist. The kinds of costs functions are varied, such as piecewise costs functions, proportional costs functions, exponential cost functions, and stochastic cost functions, etc. Since the acceptance set on the cost functions is nonconvex, the corresponding risk function must be redefined. This paper mainly studies how to define the non-cash risk measure and the corresponding risk acceptable set when the costs functions are complicated. 
Non-cash risk measure provides a direct path from unacceptable positions towards the acceptance set. It uses not only cash but also other kinds of assets to adjust the position and return the smallest amount of assets which has to be changed into an eligible quota such that the new position becomes acceptable.

The organization of this paper is as follows. In Section 2, some terminologies and the reasons to propose this new risk measure are stated. In Section 3, first the definition of the non-cash risk measure is given, then some properties of the new measure are studied, next the convex extension of the nonconvex risk measure is given and the link between them is studied at last. Section 4 gives some examples of how to calculate the non-cash risk measure. Finally, concluding remarks are given in Section 5.

\section{Preliminaries}

Coherent risk measures are the foundation of the modern risk measure theory. Artzner et al. (1999) [5] define them by the following set of axioms. A risk measure $\rho_{0}$ is called coherent if it satisfies the following axioms:

Monotonicity: If $x \geq y$, then $\rho(x) \leq \rho(y)$.

Translation invariance (cash-additivity): For $m \in \mathbb{R}$, there exists $\rho_{0}(x+m)=\rho_{0}(x)-m$,

Positive Homogeneity: $\forall \lambda \geq 0, \rho(\lambda x)=\lambda \rho(x)$, and

Subadditivity: $\rho(X+Y) \leq \rho(X)+\rho(Y)$.

Besides, traditional coherent risk measures also satisfy convexity.

Convexity: for $\lambda \in[0,1]$, there exists $\rho_{0}(\lambda x+(1-\lambda) y) \leq \lambda \rho_{0}(x)+(1-\lambda) \rho_{0}(y)$.

Interestingly, the axioms form a canonical connection between risk measures and acceptance sets. The proposition below is also given by Artzner et al. (1999) [5].

Proposition 1. (the relationship of $\rho_{A}$ and $A_{\rho}$ ) The acceptance set $A_{\rho}$ defined by any monetary risk measure $\rho: X \rightarrow \mathbb{R}$ is as follows:

$$
A_{\rho}=\{x \in X \mid \rho(x) \leq 0\}
$$

The acceptance set defined above has the property:

If $\rho(x)$ is positive homogeneous, then $A_{\rho}$ is a cone, and if $\rho(x)$ is a convex function, then $A_{\rho}$ is a convex.

On the other hand, each acceptance set $A$ defines a monetary risk measure $\rho_{A}$ as follows:

$$
\rho_{A}(x)=\inf \{m \in \mathbb{R} \mid x+m \cdot r \in A\},
$$

where $r$ is the risk-free rate in the market.

Similarly, if $A_{\rho}$ is a cone, then $\rho(x)$ satisfies the property of positive homogeneity. And if $A_{\rho}$ is a convex, then $\rho(x)$ satisfies the convexity property. That means $\rho_{A_{\rho}}=\rho$ and $A \subseteq A_{\rho_{A}}$. The equation $A=A_{\rho_{A}}$ holds if the set $A$ is closed. This dual representation proposition was proofed by Artzner et al. (1999) [5].

From the property, the nature of measure is correspondent with the acceptable set. When the set is a cone, the measure is positive homogeneous. When the acceptable set is convex, the measure is convex. So if the market is frictional, then the acceptable set could be nonconvex. The risk measure derived from the set should be redefined instead of the coherent risk measures.

Sometimes it is inappropriate to measure the risk of a position with monetary measures. For example, if there is a position represented as $x=(0,100,0)$, each component devoting cash, stock $S$, and the at-the-money put option on the stock $S$. If the risk of the new position is required to be half, then the monetary method would change the position to $x_{1}=(100,100,0)$, meaning 100 cash added. However, if it is not limited to cash only to adjust this position, then the best adjustment is $x_{2}=(0,100, p)$, meaning a put option with market price $p$ is used to hedge the risk. In this case, the risk of the stock can be offset by a little cost if the risk hedging method is appropriate. Therefore, a new risk measure which provides a direct path from the unacceptable positions towards the acceptance set 
is defined. Non-cash risk measure uses cash and other kinds of assets to adjust the position and return optimal solution of the adjustment path.

\section{Non-Cash Risk Measure on Nonconvex Sets}

This part first shows why the acceptable sets in the real world may be nonconvex. Then the non-cash risk measure is introduced. Some properties of the new measure are also studied. Next the extension of the nonconvex risk function is given. At last the relationship of this extension and the initial non-cash risk measure function is discussed.

\subsection{Introductions of Non-Cash Risk Measure}

Given the probability space $(\Omega, F, P)$ and denote by $\aleph$ the sets of the random variable $X: \Omega \mapsto \mathbb{R}$. The random variable represents the discounted net value of an investment strategy defined on the probability space. The definition of $\Omega$ is the set of all possible natural states. $X(\omega)$ is the value of the position when the state is $\omega \in \Omega$.

An acceptable set of the risk measure is defined as a set of portfolios that do not require any monetary adjustments, that is $A_{\rho}=\{x \in X \mid \rho(x) \leq 0\}$. Compared with the traditional acceptable sets of risk measures, there are friction costs in this model, which is represented as

$$
\cos t(x)=\left(\operatorname{cost}\left(x_{1}\right), \cos t\left(x_{2}\right), \cdots, \operatorname{cost}\left(x_{n}\right)\right)
$$

where $\cos t\left(x_{i}\right)$ represents the transaction costs of the $i$ th asset.

The risk measure in the friction market is defined as $\rho^{\prime}(x)=\rho_{0}(x)+\cos t(x)$, where $\rho_{0}(x)$ is a coherent risk measure in the frictionless market. This formula means the risk of the position is contained by the cash added to make it acceptable and the extra cost of purchasing the position. Then the corresponding acceptable set derived from $\rho^{\prime}$ by Formula (1) may not be convex because of the unknown cost functions.

We define a portfolio $x=\left(x_{1}, x_{2}, \cdots, x_{n}\right)$ in which each component represents the amount of investment in different types of assets. The $\mathrm{n}$ represents the total number of assets. Given tacit consent to $x \leq x+m * e_{i}, i=1,2, \cdots, n, m \geq 0, e_{i}$ is the unit vector $(0, \cdots, 0,1,0, \cdots 0)$, that means, if any amount of the component increases, the total risk of the portfolio becomes smaller.

In this paper, a new concept: non-cash risk measure is proposed. This measure uses all kinds of assets in the market to adjust the position. While avoiding the problem of cash limitations, non-cash risk measure gives an optimal solution of the adjusted position.

The definition of the non-cash risk measure is defined by the distance from the effective boundary of the portfolio to the acceptable set. Using the definition in Vakili J. (2017) [8] for reference, the non-cash risk measure is defined as follows.

$$
\rho(x)=\min _{y \in A}\left(\operatorname{dist}_{L_{\|\cdot\|}^{p}}(x, y)\right)=\min _{y \in \partial A}\left(\operatorname{dist}_{L_{\|\cdot\|}^{p}}(x, y)\right),
$$

where

$$
L^{p}(x)=\left\{\begin{array}{l}
\sum_{i=1}^{n}\left|x_{i}\right|, p=1 \\
\left(\sum_{i=1}^{n}\left|x_{i}\right|^{p}\right)^{\frac{1}{p}}, 1<p<+\infty \\
\max \left(\left|x_{i}\right|\right), p=+\infty
\end{array}\right.
$$

In this article, denote

$$
\operatorname{dist}(x, A)=\operatorname{dist}_{L_{\|\cdot\|}^{1}}(x, A)=\min _{y \in A}\left(\operatorname{dist}_{L_{\|\cdot\|}^{1}}(x, y)\right),
$$




$$
\operatorname{dist}(x, \partial A)=\operatorname{dist}_{L_{\|\cdot\|}^{1}}(x, \partial A)=\min _{y \in \partial A}\left(\operatorname{dist}_{L_{\|\cdot\|}^{1}}(x, y)\right)
$$

Then the corresponding risk measure can be simplified to

$$
\rho(x)=\min \left(\sum_{i=1}^{n}\left|y_{i}-x_{i}\right|\right)
$$

where $x=\left(x_{1}, x_{2}, \ldots, x_{n}\right) \notin A, y=\left(y_{1}, y_{2}, \ldots, y_{n}\right) \in \partial A$.

This measure indicates that if the position $x$ is outside the acceptable set, there exists a point $y$ in the acceptable set such that the distance from the point $x$ to the acceptable point $y$ can be regarded as a risk-adjusted method. The amount of asset adjustment required by this method can be expressed by $L^{1}$ norm. The measure defined in this paper requires finding the distance between $x$ and points in acceptable sets, which is the distance of position $x$ from the efficient boundary of the acceptable sets. The non-cash risk measure solves actually an optimization problem and gives the optimal solution to adjust the initial position. The risk of the position is defined by the least amount of assets adjusted to make the position acceptable.

Through the definition of $\rho(x)$ by Formula (8), it can be easily figured out that $\forall x \in X$, $\rho(x) \leq \rho_{0}(x)$, because

$$
\rho(x)=\min _{y \in A}\left(\sum_{i=1}^{n}\left|y_{i}-x_{i}\right|\right) \leq \min _{y \in A}\left|y_{1}-x_{1}\right| \leq \inf \left\{m \in \mathbb{R} \mid x+m e_{1} \in A\right\} .
$$

This inequality shows that the risk hedging method given by the non-cash risk measure is better than that given by the coherent risk measure.

If $x^{*}$ is the optimal solution of the optimization problem $\rho(x)=\min \left(\sum_{i=1}^{n}\left|y_{i}-x_{i}\right|\right)$, then the moving path along the directions of axes from $x$ to $x^{*}$ is the best solution to transfer the risky portfolio to the acceptable sets. The solution uses the least amount of assets $\sum_{i=1}^{n}\left|x_{i}^{*}-x_{i}\right|$.

\subsection{Properties of Non-Cash Risk Measure}

First we check if the non-cash risk measure still satisfies the four axioms defining coherent risk measures.

Monotonicity: If $x \geq y$, then $\rho(x) \leq \rho(y)$. Through the definition of the portfolio above, the measurement of non-cash risk also satisfies this property.

Cash-additivity: For every constant $m \in \mathbb{R}$, there exists $\rho_{0}(x+m)=\rho_{0}(x)-m$.

Cash-additivity is also called translation invariance. This axiom means that if cash is added to an asset, the reduction in risk is equal to the increase in cash. Because the risk measure in this paper is not based on cash adjustment only, this axiom is not always true in the risk measure defined in this paper.

When the marginal risk reduction in the portfolio is optimal for cash, it can be determined that the non-cash risk measurement satisfies the translation invariance.

Proposition 2. If $e_{1} \geq e_{2} \geq \cdots \geq e_{n}$, that is the same as $\rho\left(e_{1}\right) \leq \rho\left(e_{2}\right) \leq \cdots \leq \rho\left(e_{n}\right)$, then the non-cash risk measure satisfies the cash-additivity. 
Proof of Proposition 2. To prove that translation invariance $\rho\left(x+m e_{1}\right)=\rho(x)-m$ is satisfied, first it is needed to check

$$
\begin{aligned}
& \rho\left(x+m e_{1}\right)=\operatorname{dist}\left(x+m e_{1}, \partial A\right)=\min \left(\left[y_{1}-\left(x_{1}+m\right)\right]+\sum_{i=2}^{n}\left|y_{i}-x_{i}\right|\right) \\
& =\left(\left[y_{1}^{1}-\left(x_{1}+m\right)\right]+\sum_{i=2}^{n}\left|y_{i}^{1}-x_{i}\right|\right)=\left(\sum_{i=1}^{n}\left|y_{i}^{2}-x_{i}\right|\right)-m \\
& =\min \left(\sum_{i=1}^{n}\left|y_{i}-x_{i}\right|\right)-m=\operatorname{dist}(x, \partial A)-m=\rho(x)-m,
\end{aligned}
$$

where $x=\left(x_{1}, x_{2}, \ldots, x_{n}\right) \notin A, y^{1}=\left(y_{1}^{1}, y_{2}^{1}, \ldots, y_{n}^{1}\right) \in \partial A$ is the optimal solution of the first optimization problem, $y^{2}=\left(y_{1}^{2}, y_{2}^{2}, \cdots, y_{n}^{2}\right) \in \partial A$ is the optimal solution of the second optimization problem.

To get

$$
\left(\left[y_{1}^{1}-\left(x_{1}+m\right)\right]+\sum_{i=2}^{n}\left|y_{i}^{1}-x_{i}\right|\right)=\left(\sum_{i=1}^{n}\left|y_{i}^{2}-x_{i}\right|\right)-m
$$

That means to proof $\sum_{i=1}^{n}\left|y_{i}^{1}-x_{i}\right|=\sum_{i=1}^{n}\left|y_{i}^{2}-x_{i}\right|$.

Next check if $y^{1}$ is the same point as $y^{2}$.

Since $y^{2}$ is the optimal solution of the second optimization problem, then if $y^{1}=y^{2}$, we have $\forall y \in A, \operatorname{dist}\left(x+m e_{1}, y^{2}\right) \leq \operatorname{dist}\left(x+m e_{1}, y\right)$. That means the proof of $\left(y_{1}^{2}-x_{1}-m\right)+\sum_{i=2}^{n}\left(y_{i}^{2}-x_{i}\right) \leq$ $\left(y_{1}-x_{1}-m\right)+\sum_{i=2}^{n}\left(y_{i}-x_{i}\right)$.

Simplify the inequality above, we have $\sum_{i=1}^{n}\left(y_{i}^{2}-x_{i}\right) \leq \sum_{i=1}^{n}\left(y_{i}-x_{i}\right)$, which is obviously true due to the definition of $y^{2}$.

Thus the proof is complete.

Positive Homogeneity: $\forall \lambda \geq 0, \rho(\lambda x)=\lambda \rho(x)$.

The non-cash risk measure does not satisfy this axiom because the cost is added to the model and the cost function may be nonlinear. For example, if $x=(B, S)$ is a portfolio, where $\cos t(B)=c_{1}$, $\cos t(S)=c_{2} S, c_{1}, c_{2}$ is a constant, then the portfolio does not satisfy the positive homogeneity.

Subadditivity: $\rho(x+y) \leq \rho(x)+\rho(y)$.

The non-cash risk measure also satisfies this axiom. The proof is as follows.

Proposition 3. If $\rho$ is a non-cash risk measure, then it satisfies $\rho(x+y) \leq \rho(x)+\rho(y)$.

Proof of Proposition 3. If $z^{1}$ is the optimal solution of $\rho(x+y)=\min _{z \in \partial A}\left(\sum_{i=1}^{n}\left|z_{i}-\left(x_{i}+y_{i}\right)\right|\right), z^{2}$ is the optimal solution of $\rho(x)=\min _{z \in \partial A}\left(\sum_{i=1}^{n}\left|z_{i}-x_{i}\right|\right), z^{3}$ the optimal solution of $\rho(y)=\min _{z \in \partial A}\left(\sum_{i=1}^{n}\left|z_{i}-y_{i}\right|\right)$. From the tacit consent $x \leq x+m * e_{i}$ given above, it is apparently that

$$
\rho(x)=\min _{y \in \partial A}\left(\sum_{i=1}^{n}\left|y_{i}-x_{i}\right|\right)=\min _{y \in A} \sum_{i=1}^{n}\left(y_{i}-x_{i}\right) .
$$


Then we have

$$
\begin{aligned}
& \rho(x)+\rho(y)=\sum_{i=1}^{n}\left(z_{i}^{2}-x_{i}\right)+\sum_{i=1}^{n}\left(z_{i}^{3}-y_{i}\right) \\
& =\sum_{i=1}^{n}\left[\left(z_{i}^{2}-x_{i}\right)+\left(z_{i}^{3}-y_{i}\right)\right] \\
& =\sum_{i=1}^{n}\left(\left(z_{i}^{2}+z_{i}^{3}\right)-\left(x_{i}+y_{i}\right)\right) .
\end{aligned}
$$

Since $\rho(x+y)=\sum_{i=1}^{n}\left(z_{i}^{1}-\left(x_{i}+y_{i}\right)\right)$, where $z^{1}$ is the optimal solution of optimization problem $\min _{z \in \partial A}\left(\sum_{i=1}^{n}\left|z_{i}-\left(x_{i}+y_{i}\right)\right|\right)$, then

$$
\begin{aligned}
& \rho(x+y)=\min _{z \in \partial A}\left(\sum_{i=1}^{n}\left|z_{i}-\left(x_{i}+y_{i}\right)\right|\right)=\sum_{i=1}^{n}\left(z_{i}^{1}-\left(x_{i}+y_{i}\right)\right) \\
& \leq \sum_{i=1}^{n}\left(\left(z_{i}^{2}+z_{i}^{3}\right)-\left(x_{i}+y_{i}\right)\right)=\rho(x)+\rho(y) .
\end{aligned}
$$

Thus the proof is finished.

Proposition 4. In addition, the non-cash risk measure also satisfies the monotonicity of the partial derivative: If $x \notin A$, then $\frac{\partial \rho(x)}{\partial x_{i}} \leq 0, \forall i=1,2, \cdots, n$.

\section{Proof of Proposition 4.}

$$
\begin{aligned}
& \frac{\partial \rho(x)}{\partial x_{i}}=\lim _{\delta \rightarrow 0} \frac{\rho\left(x+\delta \cdot e_{i}\right)-\rho(x)}{\delta} \\
& =\operatorname{dist}\left(x+\delta \cdot e_{i}, \partial A\right)-\operatorname{dist}(x, \partial A) \leq 0 .
\end{aligned}
$$

Let proof by contradiction.

If $\operatorname{dist}\left(x+\delta \cdot e_{i}, \partial A\right)-\operatorname{dist}(x, \partial A)>0$, that is $\operatorname{dist}\left(x+\delta \cdot e_{i}, \partial A\right)>\operatorname{dist}(x, \partial A)$, since $x \leq x+m * e_{i}$, $i=1,2, \cdots, n, m \geq 0$, then $\operatorname{dist}\left(x+\delta \cdot e_{i}, \partial A\right) \leq \operatorname{dist}(x, \partial A)$ is contradictory to the assumption. However, it is found that the risk measure does not satisfy convexity. A counterexample of convexity is given below. If $x=0, y \in \partial A$, since the acceptable set is a nonconvex set, there exists $\lambda \in[0,1]$, such that $\lambda x+(1-\lambda) y \notin A$. In this condition, there exists $\rho(\lambda x+(1-\lambda) y)>0$, contradictory to $\lambda \rho(x)+(1-\lambda) \rho(y)=0$.

\subsection{Extension of Non-Cash Risk Measure}

Since the risk measure is not a convex function and solves an optimization problem, first a convex risk measure function derived from the extension of the risk measure is given. Then the relationship between the convex risk measure and the original non-cash risk measure is studied. In reference to the use of the concave hull in Lepinette et al. (2015) [9], we define the convex risk measure as follows:

$$
\tilde{\rho}(x)=\inf \left\{\sum_{i=1}^{n} \lambda_{i} \rho\left(x_{i}\right): \lambda_{i} \geq 0, \sum_{i=1}^{n} \lambda_{i} x_{i}=x\right\},
$$

where $\tilde{\rho}(x)$ is the biggest convex hull of $\rho(x)$.

Obviously, the measure function has the following properties.

1. $\tilde{\rho}(x) \leq \rho(x)$,

2. Subadditivity: $\tilde{\rho}(X+Y) \leq \tilde{\rho}(X)+\tilde{\rho}(Y)$,

3. Convexity: $\tilde{\rho}(\lambda x+(1-\lambda) y)=\lambda \tilde{\rho}(x)+(1-\lambda) \tilde{\rho}(y), \lambda \in[0,1]$. 
The following studies the relationship of $\tilde{\rho}(x)$ and $\rho(x)$.

Lemma 1. Denote $g(x)=D_{+\infty} \rho(x)=\lim _{\lambda \rightarrow+\infty} \frac{\rho(\lambda x)}{\lambda}$, then $g(x)$ is a convex function.

Proof of Lemma 1. When $\lambda \geq 1$, we have

$$
\begin{aligned}
& g(u x)=D_{+\infty} \rho(u x)=\lim _{\lambda \rightarrow+\infty} \frac{\rho(\lambda \cdot u x)}{\lambda}=\lim _{\lambda \rightarrow+\infty} u \cdot \frac{\rho(\lambda u \cdot x)}{\lambda u} \\
& =u \cdot \lim _{\lambda \rightarrow+\infty} \frac{\rho(\lambda u \cdot x)}{\lambda u}=u \cdot \lim _{\beta \rightarrow+\infty} \frac{\rho(\beta x)}{\beta}=u \cdot D_{+\infty} \rho(x) .
\end{aligned}
$$

That is $g(u x)=u g(x)$, which implies that $g(x)$ satisfies the linear condition.

Denote $\mu>0, \eta>0$ and $\lambda>0$ such that $\lambda \geq \mu \vee \eta$, then $\frac{\lambda}{\mu} \geq 1, \frac{\lambda}{\eta} \geq 1$.

Define $\rho(\lambda(x+y))=\min _{x^{*} \in \partial A}\left(\sum_{i=1}^{n}\left|x_{i}^{*}-\lambda\left(x_{i}+y_{i}\right)\right|\right)$ has the optimal solution of $x^{c}=\left(x_{1}^{c}, x_{2}^{c}, \cdots, x_{n}^{c}\right)$, and $\rho(\mu x)=\min _{x^{*} \in \partial A}\left(\sum_{i=1}^{n}\left|x_{i}^{*}-\mu x_{i}\right|\right)$ has the optimal solution which is $x^{a}=\left(x_{1}^{a}, x_{2}^{a}, \cdots, x_{n}^{a}\right)$, and $\rho(\mu y)=\min _{x^{*} \in \partial A}\left(\sum_{i=1}^{n}\left|x_{i}^{*}-\mu y_{i}\right|\right)$ has the optimal solution of $x^{b}=\left(x_{1}^{b}, x_{2}^{b}, \cdots, x_{n}^{b}\right)$, then

$$
\begin{aligned}
& \rho(\lambda(x+y))=\sum_{i=1}^{n}\left[x_{i}^{c}-\lambda\left(x_{i}+y_{i}\right)\right] \\
& \leq \sum_{i=1}^{n}\left[\left(\frac{\lambda}{\mu} \cdot x_{i}^{a}+\frac{\lambda}{\eta} \cdot x_{i}^{b}\right)-\left(\lambda x_{i}+\lambda y_{i}\right)\right] \\
& =\sum_{i=1}^{n}\left[\left(\frac{\lambda}{\mu} \cdot x_{i}^{a}-\lambda x_{i}\right)+\left(\frac{\lambda}{\eta} \cdot x_{i}^{b}-\lambda y_{i}\right)\right] \\
& =\sum_{i=1}^{n} \frac{\lambda}{\mu}\left(x_{i}^{a}-\mu x_{i}\right)+\sum_{i=1}^{n} \frac{\lambda}{\eta}\left(x_{i}^{b}-\mu y_{i}\right) \\
& =\frac{\lambda}{\mu} \sum_{i=1}^{n}\left(x_{i}^{a}-\mu x_{i}\right)+\frac{\lambda}{\eta} \sum_{i=1}^{n}\left(x_{i}^{b}-\mu y_{i}\right) \\
& =\frac{\lambda}{\mu} \cdot \rho(\mu x)+\frac{\lambda}{\eta} \cdot \rho(\eta y) .
\end{aligned}
$$

So that

$$
\begin{aligned}
& \rho(\lambda(x+y))=\sum_{i=1}^{n}\left[x_{i}^{c}-\lambda\left(x_{i}+y_{i}\right)\right] \\
& \leq \sum_{i=1}^{n}\left[\left(\frac{\lambda}{\mu} \cdot x_{i}^{a}+\frac{\lambda}{\eta} \cdot x_{i}^{b}\right)-\left(\lambda x_{i}+\lambda y_{i}\right)\right] \\
& =\sum_{i=1}^{n}\left[\left(\frac{\lambda}{\mu} \cdot x_{i}^{a}-\lambda x_{i}\right)+\left(\frac{\lambda}{\eta} \cdot x_{i}^{b}-\lambda y_{i}\right)\right] \\
& =\sum_{i=1}^{n} \frac{\lambda}{\mu}\left(x_{i}^{a}-\mu x_{i}\right)+\sum_{i=1}^{n} \frac{\lambda}{\eta}\left(x_{i}^{b}-\mu y_{i}\right) \\
& =\frac{\lambda}{\mu} \sum_{i=1}^{n}\left(x_{i}^{a}-\mu x_{i}\right)+\frac{\lambda}{\eta} \sum_{i=1}^{n}\left(x_{i}^{b}-\mu y_{i}\right) \\
& =\frac{\lambda}{\mu} \cdot \rho(\mu x)+\frac{\lambda}{\eta} \cdot \rho(\eta y) .
\end{aligned}
$$


When $\mu, \eta \rightarrow+\infty, g(x+y) \leq g(x)+g(y)$ is also sub- additive. Then

$$
g(\lambda x+y) \leq g(\lambda x)+g((1-\lambda) y)=\lambda g(x)+(1-\lambda) g(y) .
$$

Which means $g(x)$ is a convex function.

Theorem 1. If a non-cash risk measure $\rho(x)$ is a measure defined on a non-convex acceptable set and $\tilde{\rho}(x)$ is a convex extension of $\rho(x)$, the following formula holds:

$$
\tilde{\rho}(x)=D_{+\infty} \rho(x)=\lim _{\lambda \rightarrow+\infty} \frac{\rho(\lambda x)}{\lambda} .
$$

Proof of Theorem 1. By Lemma 1 and the definition of $\tilde{\rho}(x)$, it is clear that $g(x) \leq \tilde{\rho}(x)$.

If $n \geq 1$, then $\tilde{\rho}_{1}(x) \leq \sum_{\mathrm{i}=1}^{n} \rho\left(\frac{x}{n}\right)=n \rho\left(\frac{x}{n}\right)$. When $n \rightarrow+\infty$, denote $\lambda=\frac{1}{n}$, then $\lambda \rightarrow 0^{+}$and we have $\tilde{\rho}(x) \leq \lim _{\lambda \rightarrow 0+} \frac{\rho(\lambda x)}{\lambda}$. When $n \rightarrow 0^{+}$, denote $\lambda=\frac{1}{n}$, then we have $\tilde{\rho}(x) \leq \lim _{\lambda \rightarrow+\infty} \frac{\rho(\lambda x)}{\lambda}$, that is $\tilde{\rho}(x) \leq g(x)$. After all, $\tilde{\rho}(x)=g(x)$.

\section{Examples}

Example 1. (Financial markets with fixed and proportional transaction costs) Consider a financial market model in which a portfolio contains two assets. The first component with price $x_{1}$ is a class of bond without any cost of buying or selling. The second component with price $x_{2}$ is the stock. The cost of the stock consists of two parts. The first part is the fixed cost cost ${ }_{\text {fix }}\left(x_{2}\right)=c$, where $c$ is a constant, that is, no matter how many transactions are made, the transaction cost will occur as long as the transaction takes place. The second part cost $_{\text {proportion }}\left(x_{2}\right)=k\left|x_{2}\right|$ is the transaction cost distributed proportionally. Where $k$ is the constant between 0 to 1 . We assume that the asset is too risky to be included in the acceptable set when the transaction cost is greater than the exchange gain. This means when $x_{1}+x_{2} \leq \operatorname{cost}_{\text {fix }}\left(x_{2}\right)+\operatorname{cost}_{\text {proportion }}\left(x_{2}\right)$, or $x_{1}+x_{2} \leq c+k\left|x_{2}\right|$, then $x=\left(x_{1}, x_{2}\right)$ is not contained in the acceptable set $A$. The corresponding non cash-risk measure is as follows:

$$
\rho\left(\left(x_{1}, x_{2}\right)\right)=1_{x_{2} \neq 0} \cdot c+k\left|x_{2}\right|-x_{1}-x_{2} .
$$

The acceptance set defined by $A=\left\{x=\left(x_{1}, x_{2}\right) \in X \mid \rho\left(\left(x_{1}, x_{2}\right)\right) \leq 0\right\}$ is showed in the Figure 1a below.

Example 2. (financial markets with proportional transaction costs and random costs) Consider a financial market model in which a portfolio contains two assets. The first component with price $x_{1}$ is a class of bond with a proportionate transaction cost cost $t_{\text {proportion }}\left(x_{1}\right)=k\left|x_{1}\right|$ where $k$ is the constant between 0 to 1 . The second component $x_{2}$ is a commodity contract, where part of the cost is the purchase cost that varies with the underlying asset value and the other part is the transaction cost that is randomly generated. We assume that the asset is too risky to be included in the acceptable set when the transaction cost is greater than the gains. So when $x_{1}+x_{2} \leq \operatorname{cost}_{\text {proportion }}\left(x_{2}\right)+\operatorname{cost}_{\text {rand }}\left(x_{2}\right)$, or $x_{1}+x_{2} \leq k\left|x_{2}\right|+$ rand $(0,1)$, then $x=\left(x_{1}, x_{2}\right) \notin A$. The corresponding non-cash risk measure is as follows:

$$
\rho\left(\left(x_{1}, x_{2}\right)\right)=k\left|x_{2}\right|+\operatorname{rand}(0,1)-x_{1}-x_{2} \text {. }
$$

The corresponding acceptance set is showed in the Figure $1 b$ below. 


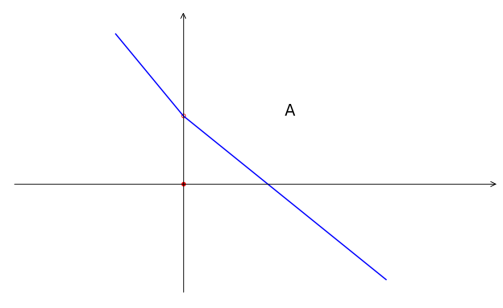

(a)

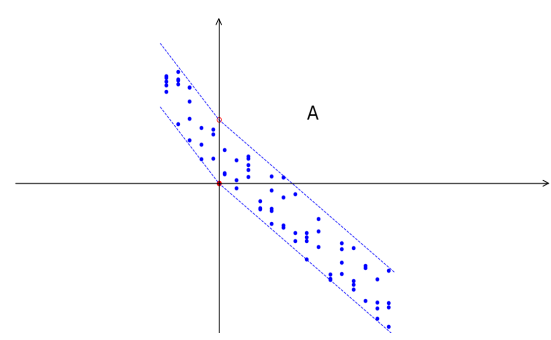

(b)

Figure 1. The corresponding sets of examples. Figure (a) shows the acceptable set with fixed costs and proportional transaction costs, Figure (b) shows the acceptable set with proportional transaction costs and stochastic costs.

\section{Conclusions}

This paper mainly focus on the corresponding risk measure when an acceptable set is a nonconvex set. By discussing the cost function, a new measure is given, which is superior to the coherent risk measure in risk adjustment. At the same time, since the non-cash risk measure is a non-convex measure and the corresponding acceptable set is also non-convex, we also discuss the extension of the non-cash risk measure and give the relationship between the extension risk measure and the non-cash risk measure.

Author Contributions: Conceptualization, C.C. and P.Z.; Methodology, C.C. and P.Z.; Validation, C.C. and P.Z.; Formal Analysis, C.C. and P.Z.; Writing-Original Draft Preparation, C.C.; Writing-Review and Editing, P.Z.; Funding Acquisition, P.Z.

Funding: This research was supported by the National Natural Science Foundation of China (No. 11871275, No. 11371194).

Conflicts of Interest: The authors declare no conflict of interest.

\section{References}

1. Markowitz, H. Portfolio Selection. J. Financ. 1952, 7, 77-91. [CrossRef]

2. Morgan, J.P. CreditMetrics, Technical Documents; J.P. Morgan Bank: New York, NY, USA, 1997.

3. Acerbi, C.; Tasche, D. On the coherence of expected shortfall. J. Bank. Financ. 2002, 26, 1487-1503. [CrossRef]

4. Frey, R.; Mcneil, A. VaR and expected shortfall in credit portfolios: Conceptual and practical insights. J. Bank. Financ. 2002, 26, 1317-1334. [CrossRef]

5. Artzner P.; Delbaen F.; Eber, J.M.; Heath, D. Coherent Measures of Risk Mathematical Finance. Math. Financ. Theory Model. Implement. 1999, 9, 203-228. [CrossRef]

6. Follmer, H.; Schied, A. Convex measures of risk and trading constraints. Financ. Stoch. 2002, 6, 429-447. [CrossRef]

7. Lepinette, E.; Tran, T. Arbitrage theory for non-convex financial market models. Stoch. Process. Their Appl. 2008, 127, 3331-3353. [CrossRef]

8. Vakili, J. New Models for Computing the Distance of DMUs to the Weak Efficient Boundary of Convex and Nonconvex PPSs in DEA. Asia-Pac. J. Oper. Res. 2017, 34, 1750035. [CrossRef]

9. Lepinette, E.; Tran, T. General financial market model defined by a liquidation value process. Stoch. Int. J. Probab. Stoch. Process. 2015, 88, 437-459. [CrossRef]

(c) 2018 by the authors. Licensee MDPI, Basel, Switzerland. This article is an open access article distributed under the terms and conditions of the Creative Commons Attribution (CC BY) license (http://creativecommons.org/licenses/by/4.0/). 